\title{
Practical Model for Real Structure and Performance of
}

\section{Heavy Vehicles}

\author{
Younès Abbassi ${ }^{1}$, Youcef Ait-Amirat ${ }^{2}$ and Rachid Outbib ${ }^{3}$ \\ 1. Department of Energy, University of Technology of Belfort-Montbéliard, Belfort 90010, France \\ 2. Department of Energy, University of Franche-Comté, Belfort 90010, France \\ 3. LSIS (Laboratoire des Sciences de l'Information et des Systèmes), University of Aix-Marseille, Marseille 13397, France
}

Received: November 14, 2014 / Accepted: December 01, 2014 / Published: February 25, 2015.

\begin{abstract}
In this paper, a new practical model for real heavy vehicle structure is developed to investigate dynamic responses under steering/acceleration or braking maneuvers. The generalized six DoFs (degrees-of-freedom) nonlinear vehicle model $M_{1}$ including longitudinal, lateral, yaw, vertical, roll and pitch dynamics is validated using the measured data reported in different studies. This model takes the CG (center of gravity) of sprung mass, unsprung mass and total vehicle mass into account. Based on this model, the effects of the inertia parameters on the vehicle dynamic responses are investigated for more comprehensive assessments of the model structure. Another nonlinear vehicle model $M_{2}$ derived from $M_{1}$ which assumes that the vehicle has a single CG as reported in literature is also developed. The dynamic responses of the vehicle model $M_{1}$ are compared with those of the model $M_{2}$ to demonstrate the performance potential of the proposed nonlinear model. The results of dynamic responses with the nonlinear vehicle model $M_{1}$ suggest that the model could offer considerable potential in realizing enhanced ride and handling performance, as well as improved roll and pitch properties in a flexible manner.
\end{abstract}

Key words: Modeling, nonlinear vehicle models, inertial parameters, heavy vehicle dynamics, ride, handling.

\section{Introduction}

Steering, braking or acceleration maneuvers of road vehicles induce generally comprehensive magnitudes of vehicle yaw, roll and pitch motion and thus the knowledge of the position of various center of gravity. During motion in particular in turn, the CG (center of gravity) of the vehicle, the CG of the unsprung mass and that of the sprung mass are different arising from dynamic load transfers due to the cornering and braking forces developed by the tires $[1,2]$. Taking into account both CG of the sprung mass and unsprung mass can give more flexibility to the vehicle and inhibit the longitudinal and lateral load transfers. To study the vehicle dynamics, a range of linear and nonlinear models of road vehicles have been reported in the

Corresponding author: Younès Abbassi, Dr. Eng., research fields: vehicles system dynamics and nonlinear dynamics and control.E-mail: younes.abbassi@ac-besancon.fr. literature for analysis of ride, handling and directional control characteristics. These models vary from simple linear models [3, 4] to complex nonlinear models $[4,5]$. Actually, the vehicle dynamics is coupled into a single dynamic model in which all elements are interconnected and act differently. This means that the vehicle is composed with different rigid bodies and elastic elements and not with a single rigid body which has only center of gravity. The different models generally consider that the vehicle has only one center of gravity G. Accordingly, the vehicle is assimilated to one solid placed firmly on the wheels. The majority of the reported studies concentrate on solving for the design conflict between the ride comforts and handling performance requirements. While the majority of studies focus more on the ride dynamics aspect ignoring the effects of inertial parameters. As a consequence of this consideration to the vehicle dynamics, the flexibility in maneuvering is altered and 
the inertial parameters such moments of inertia can be directly reduced. Therefore, yaw, roll and pitch increase that will have a direct impact on the vehicle handling $[6,7]$, braking and traction performance [8]. These effects are well reported in the literature and the above references are by no means exhaustive. In the absence of real dynamic model which takes into account both the inertial parameters of sprung mass and unsprung mass and total vehicle mass, the discrepancies between the real vehicle design and its model dynamic become large. These discrepancies affect such active control system which often used fixed values for the inertial parameters that lie somewhere between the maximum and minimum expected values.

In this works, the main goal is to show how the vehicle motion is affected when we consider only one $\mathrm{CG}$ for the total vehicle mass. It also shows the stability and flexibility properties results when we consider simultaneously the CG of sprung mass $G_{s}$ and the CG of unsprung mass $G_{u}$. Moreover, the roll and pitch characteristics of the vehicle suspension are inhibited as well as the lateral and longitudinal load transfers. As a result, the suspension could provide adequate attenuation of the road-induced vibration.

This paper is organized as follows: Section 2 develops equations of motion for the present works and derives two nonlinear vehicle models $M_{1}$ and $M_{2}$ suitable for the present study; Section 3 discusses the simulation results and the validation of the proposed vehicle model $M_{1}$; finally, Section 4 gives conclusions.

\section{Nonlinear Vehicle Models}

A range of linear and nonlinear models of road vehicles are reported in the literature for vehicles analysis, handling and control $[9,10]$. These models consider that the vehicle has only one CG as shown in Fig. 3. This means that the distances $c_{p}$ and $c_{u}$ and the height $h_{u}$ are zero, i.e., $c_{p}=c_{u}=h_{u}=0$ and $h_{p}=h$. Therefore, the roll, pitch and yaw moments of inertia of the total vehicle mass: $I_{x}, I_{y}$ and $I_{z}$ are equal to those of the only sprung mass: $I_{x s}, I_{y s}$ and $I_{z s}$, i.e., $I_{x}=I_{x s}, I_{y}=I_{y s}$ and $I_{z}=I_{z s}$. It results that many geometrical quantities are not taken into account and can thus influence the dynamic behavior of the vehicle. These geometric quantities appear when we consider that the vehicle is composed of two bodies: sprung mass and unsprung mass and each has its own center of mass.

\subsection{Nonlinear Vehicle Model: First Model $M_{1}$}

In this study, a generalized six DoFs (degrees-of-freedom) nonlinear model of a two axis vehicle, incorporating sprung and unsprung mass configurations, is developed to investigate dynamic responses to steering and braking/acceleration inputs as well as excitations arising from road roughness or crosswinds. Moreover, this nonlinear vehicle model offers the essential vehicle flexibility and may thus be applicable for the studies of rollover prevention strategies.

The proposed model shown in Fig. 1 includes six DOFs, three frame Cartesian displacements and three Euler angles: roll angle $\theta$, pitch angle $\phi$ and yaw angle $\psi$. The rates of the Euler angles (roll rate $V_{\theta}$, pitch rate $V_{\phi}$ and yaw rate $V_{\psi}$ ) are derived from Ref. [11] and can be written as:

$$
\left[\begin{array}{c}
\dot{\theta} \\
\dot{\phi} \\
\dot{\psi}
\end{array}\right]=\left[\begin{array}{c}
V_{\theta}+\phi V_{\psi} \\
V_{\phi}-\theta V_{\psi} \\
V_{\psi}+\theta V_{\psi}
\end{array}\right]
$$

where, $\dot{\theta}, \dot{\phi}$ and $\dot{\psi}$ are the roll, pitch and yaw angular velocities. $V_{\theta}, V_{\phi}$ and $V_{\psi}$ are roll, pitch and yaw velocities, respectively.

The total vehicle mass $M$ has a center of mass $\mathrm{G}$ and is composed of sprung mass $M_{s}$ and unsprung mass $M_{u}$. The sprung mass could rotate about its roll axis, while the unsprung mass $M_{u}$ is supposed to be the mass of a one solid which contains the total mass of the wheels and others solids attached to the bottom of the suspension.

The coordinates of the CG of total vehicle mass, sprung mass and unsprung mass in the vehicle reference frame $\left(x_{0}, y_{0}, z_{0}\right)$ are $(0,0, h),\left(-c_{u}, 0, h_{u}\right)$ 


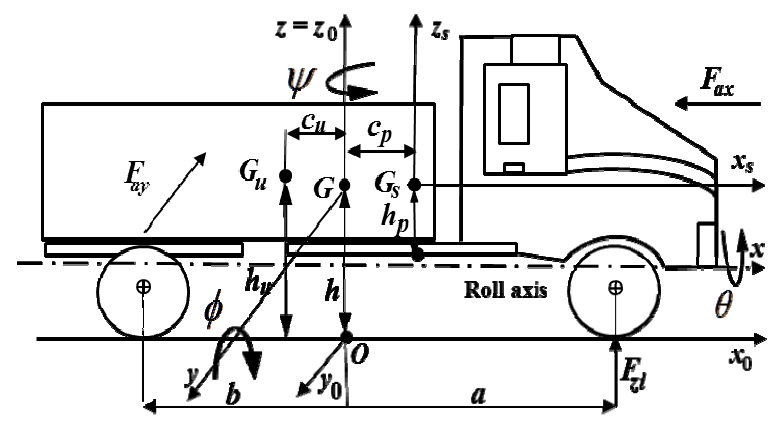

Fig. 1 Heavy vehicle structure for $\operatorname{model} M_{1}$.

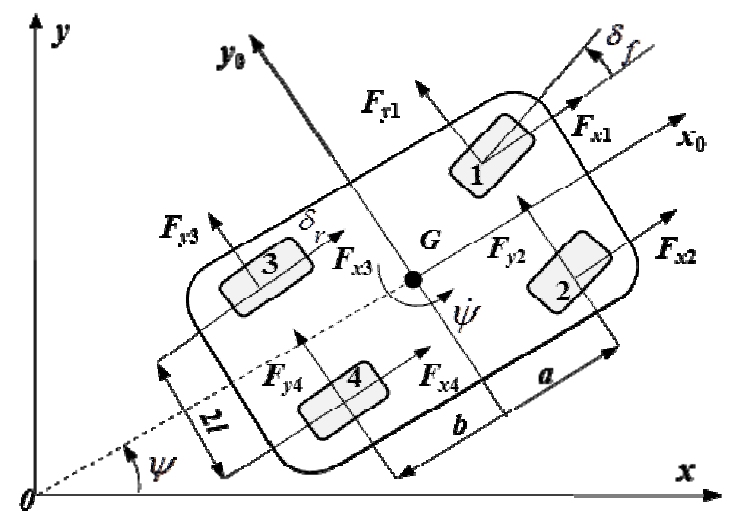

Fig. 2 Nonlinear braking/acceleration and cornering forces.

and $\left(c_{u}, 0, h_{u}\right)$, respectively. The exact position of the CG of unsprung mass is generally determined by the vehicle constructor. The roll angle and the moments of inertia of the unsprung mass are usually small and thus could be neglected. The kinetic and potential energy of the unsprung mass depend generally on the total mass of wheels for simplicity, the unsprung mass $M_{u}$ can be assumed to be the sum of the total mass of the wheels $M_{w i(i=1, \ldots, 4)}$ and the front and rear spindle mass $\left(M_{s p f}\right.$, $\left.M_{s p r}\right)$.

$$
M_{u}=M_{s p f}+M_{s p r}+\sum_{i}^{4} M_{w i}
$$

The front-wheel steering input $\delta_{f}$ is assumed to be identical for both the front wheels, while the rear-wheel steering input $\delta_{r}$ could also be conveniently included in the vehicle model for analysis of vehicles with 4WS (four-wheel steering).

The nonlinear braking, acceleration $F_{x i}$ and cornering forces $F_{y i}$ shown in Fig. 2 developed by the tires [12], are evaluated as functions of the normal forces, slip angle and slip ratio using the Magic Formula tire model which is widely used as a leading tire model $[13,14]$. The magic formula tire model is used to derive braking/acceleration, cornering forces and aligning moment developed by a tire, as a function of the slip angle $\alpha_{i},(i=1, \ldots, 4)$, and the normal tire forces $F_{z i}$. By assuming small steering angles, the slip angle used in the tire model $M_{1}$ is can be expressed as

$$
\begin{aligned}
& \alpha_{1}=\delta_{f}-\frac{V_{y}-\theta V_{z}+\left(a+c_{u}-c_{u}\right) V_{\psi}}{V_{x}+\phi V_{z}+l V_{\psi}} \\
& \alpha_{2}=\delta_{f}-\frac{V_{y}-\theta V_{z}+\left(a+c_{u}-c_{u}\right) V_{\psi}}{V_{x}+\phi V_{z}-l V_{\psi}} \\
& \alpha_{3}=\delta_{r}-\frac{V_{y}-\theta V_{z}-\left(b+c_{p}-c_{u}\right) V_{\psi}}{V_{x}+\phi V_{z}+l V_{\psi}} \\
& \alpha_{4}=\delta_{r}-\frac{V_{y}-\theta V_{z}-\left(b+c_{p}-c_{u}\right) V_{\psi}}{V_{x}+\phi V_{z}-l V_{\psi}}
\end{aligned}
$$

where, $\delta_{f}$ and $\delta_{r}$ are the front and rear steering angles, respectively. $V_{x}, V_{y}$ and $V_{z}$ are longitudinal, lateral and vertical velocities of the sprung mass, respectively. $a, b$ represent the distance from $G$ to front and rear wheel axes. The distance $l$ is the half gauge of the vehicle. $c_{p}$ is the distance from $G$ to $G_{s}$ in $x$ direction. $c_{u}$ is the distance from $G$ to $G_{u}$ in $x$ direction. $V_{\psi}$ is the yaw velocity.

The excitations arising from crosswinds are incorporated in the equations of motion by an equivalent longitudinal force $F_{a x}$ and equivalent lateral force $F_{a y}$ applied to the vehicle CG [15].

$$
\begin{aligned}
& F_{a x}=-\frac{1}{2} S_{x} \rho c_{a x} V_{x}^{2}=-k_{a x} V_{x}^{2} \\
& F_{a y}=-\frac{1}{2} S_{y} \rho c_{a y} V_{y}^{2}=-k_{a y} V_{y}^{2}
\end{aligned}
$$

where, $S$ is the aerodynamic area (shadow area); $c_{a}$ is the aerodynamic coefficient and $\rho$ is the air density $\left(\mathrm{kg} \cdot \mathrm{m}^{-3}\right)$, default $=1.25$.

The equations of motion for the sprung $M_{s}$ and unsprung masse $M_{u}$ can be obtained by using Lagrangian dynamics, and the nonlinear vehicle model $M_{1}$ can be written as: 


$$
\left\{\begin{aligned}
M \dot{V}_{x}= & M V_{\psi} V_{y}-M_{s} h V_{\psi} V_{\theta}-A_{h} \dot{V}_{\phi} \\
& +F_{x f} \cos \delta_{f}+F_{x r} \cos \delta_{r}-F_{y f} \sin \delta_{f} \\
& -F_{y r} \sin \delta_{r}-k_{a x} V_{x}^{2} \\
M \dot{V}_{y} & =-M V_{\psi} V_{x}+M_{s} h \dot{V}_{\theta}-A_{c} \dot{V}_{\psi} \\
& +F_{x f} \sin \delta_{f}+F_{x r} \sin \delta_{r}+F_{y f} \cos \delta_{f} \\
& +F_{y r} \cos \delta_{r}-k_{a y} V_{y}^{2} \\
M_{s} \dot{V}_{z} & =M_{s} g-\left(K_{f}+K_{r}\right) z-\left(B_{f}+B_{r}\right) V_{z} \\
& +\left(a K_{f}-b K_{r}\right) \phi+\left(a B_{f}-b B_{r}\right) V_{\phi} \\
& -M_{s} \phi \dot{V}_{x} \\
I_{x} \dot{V}_{\theta}= & M_{s} h_{p}\left(\dot{V}_{y}+V_{\psi} V_{x}\right)+A_{y z} V_{\psi} V_{\phi}+A_{y z} V_{\psi} V_{\phi} \\
+ & I_{x z s} \dot{V}_{\psi}-\left(l^{2} K_{f}+l^{2} K_{r}+B_{a f}+B_{a r}\right) \theta \\
- & \left(l^{2} B_{f}+l^{2} B_{r}\right) V_{\theta}-M_{s} g h \theta \\
- & \left(a^{2} K_{f}+b^{2} K_{r}-M_{s} g h\right) \phi \\
& -\left(a^{2} B_{f}+b^{2} B_{r}\right) V_{\phi} \\
I_{y} \dot{V}_{\phi} & =M_{s} c_{p} \dot{V}_{z}-A_{h}\left(\dot{V}_{x}-V_{\psi} V_{y}\right)+A_{z x} V_{\psi} V_{\theta} \\
& -\left(a^{2} K_{f}+b^{2} K_{r}-M_{s} g h\right) \phi \\
& -\left(a^{2} B_{f}+b^{2} B_{r}\right) V_{f}+\left(a B_{f}-b B_{r}\right) V_{z} \\
& +\left(a K_{f}-b K_{r}\right) z \\
\dot{I}_{z} \dot{V}_{\psi}= & A_{x y} V_{\theta} V_{\phi}+a\left(F_{x f} \sin \delta_{f}+F_{y f} \cos \delta_{f}\right) \\
& -b\left(F_{x r} \sin \delta_{r}+F_{y r} \cos \delta_{r}\right) \\
+ & l\left(\Delta F_{x f} \cos \delta_{f}-\Delta F_{y f} \sin \delta_{f}\right) \\
+ & l\left(\Delta F_{x r} \cos \delta_{r}-\Delta F_{y r} \sin \delta_{r}\right)+I_{x z s} \dot{V}_{\theta}
\end{aligned}\right.
$$

where,

$$
\begin{gathered}
\left\{\begin{array} { l } 
{ A _ { h } = M _ { s } h _ { p } + M _ { u } h _ { p } } \\
{ A _ { c } = M _ { s } c _ { p } + M _ { u } c _ { u } } \\
{ A _ { y z } = I _ { y s } - I _ { z s } } \\
{ A _ { z x } = I _ { z s } - I _ { x s } }
\end{array} \text { and } \left\{\begin{array}{c}
I_{x}=I_{x s}+M_{s} h_{p}^{2} \\
I_{y}=I_{y s}+M_{s}\left(h_{p}^{2}+c_{p}^{2}\right) \\
+M_{u}\left(h_{u}^{2}+c_{u}^{2}\right) \\
I_{z}=I_{z s}+M_{s} c_{p}^{2}+M_{u} c_{u}^{2}
\end{array}\right.\right. \\
\left\{\begin{array} { l } 
{ F _ { x f } = F _ { x 1 } + F _ { x 2 } } \\
{ F _ { x r } = F _ { x 3 } + F _ { x 4 } } \\
{ \Delta F _ { x f } = F _ { x 2 } - F _ { x 1 } } \\
{ \Delta F _ { x r } = F _ { x 4 } - F _ { x 3 } }
\end{array} \text { and } \left\{\begin{array}{l}
F_{y f}=F_{y 1}+F_{y 2} \\
F_{x r}=F_{y 3}+F_{y 4} \\
\Delta F_{y f}=F_{y 2}-F_{y 1} \\
\Delta F_{y r}=F_{y 4}-F_{y 3}
\end{array}\right.\right.
\end{gathered}
$$

In the developed model $M_{1}$, the roll and pitch inertias of unsprung mass are not included for simplicity reason. The notations $V_{x}, V_{y}$ and $V_{z}$ are longitudinal, lateral and vertical velocities of the vehicle, respectively. $V_{\theta}, V_{\phi}$ and $V_{\psi}$ are the roll, pitch and yaw rate. $g$ is acceleration due to gravity and $a, b$ represent the distance from $G$ to front and rear wheel axes. The distance $l$ is the half gauge of the vehicle. $B_{a f}$ and $B_{a r}$ are stiffness constants of the front and rear anti-roll bars, respectively. $K_{f}$ and $K_{r}$ are stiffness constants of the front and rear suspensions, respectively. $B_{f}$ and $B_{r}$ are stiffness constants of the front and rear damping, respectively. $I_{x z}$ and $I_{x z s}$ are pitch-plane cross moment of inertia of total vehicle mass and sprung mass, respectively.

\subsection{Nonlinear Vehicle Model: Second Model M}

To investigate the effects of the inertial parameters on the vehicle stability and maneuvering capabilities, we consider a second model $M_{2}$ usually used in literature as proposed in Refs. [16, 17]. This model is deduced from model $M_{1}$ when the CG of sprung and unsprung mass are merged with the CG of the total vehicle mass as shown in Fig. 3. This means in model $M_{2}$, we have:

$$
G=G_{s}=G_{u} \Rightarrow\left\{\begin{array}{l}
h=h_{p}=h_{u} \\
M=M_{s}+M_{u} \\
c_{p}=c_{u}=0
\end{array}\right.
$$

Then, the parameters given in Eq. (5) become in $M_{2}$ :

$$
\left\{\begin{array} { l } 
{ A _ { h } = M h , \mathrm { A } _ { c } = 0 } \\
{ A _ { y z } = I _ { y } - I _ { z } } \\
{ A _ { z x } = I _ { z } - I _ { x } }
\end{array} \text { and } \left\{\begin{array}{l}
I_{x}=I_{x s}+M h^{2} \\
I_{y}=I_{y s}+M h^{2} \\
I_{z}=I_{z s}
\end{array}\right.\right.
$$

So, we obtain:

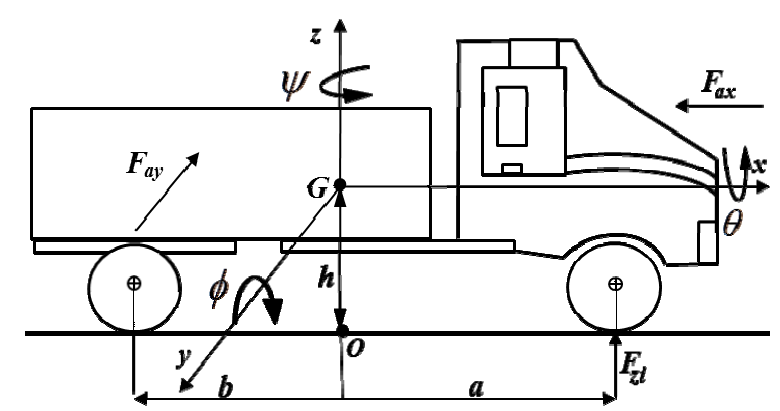

Fig. 3 Heavy vehicle structure for $\operatorname{model} M_{2}$. 


$$
\left\{\begin{aligned}
M \dot{V}_{x}= & M\left(V_{\psi} V_{y}-h V_{\psi} V_{\theta}-h \dot{V}_{\phi}\right) \\
& +F_{x f} \cos \delta_{f}+F_{x r} \cos \delta_{r}-F_{y f} \sin \delta_{f} \\
& -F_{y r} \sin \delta_{r}-k_{a x} V_{x}^{2} \\
M \dot{V}_{y} & =-M V_{\psi} V_{x}+M_{s} h \dot{V}_{\theta} \\
& +F_{x f} \sin \delta_{f}+F_{x r} \sin \delta_{r}+F_{y f} \cos \delta_{f} \\
& +F_{y r} \cos \delta_{r}-k_{a y} V_{y}^{2} \\
M_{s} \dot{V}_{z} & =M_{s} g-\left(K_{f}+K_{r}\right) z-\left(B_{f}+B_{r}\right) V_{z} \\
& +\left(a K_{f}-b K_{r}\right) \phi+\left(a B_{f}-b B_{r}\right) V_{\phi} \\
& -M_{s} \phi \dot{V}_{x} \\
I_{x} \dot{V}_{\theta}= & M_{s} h_{p}\left(\dot{V}_{y}+V_{\psi} V_{x}\right)+A_{y z} V_{\psi} V_{\phi}+A_{y z} V_{\psi} V_{\phi} \\
+ & I_{x z} \dot{V}_{\psi}-\left(l^{2} K_{f}+l^{2} K_{r}+B_{a f}+B_{a r}\right) \theta \\
& -\left(l^{2} B_{f}+l^{2} B_{r}\right) V_{\theta}-M_{s} g h \theta \\
& -\left(a^{2} K_{f}+b^{2} K_{r}-M_{s} g h\right) \phi \\
& -\left(a^{2} B_{f}+b^{2} B_{r}\right) V_{\phi} \\
I_{y} \dot{V}_{\phi} & =-A_{h}\left(\dot{V}_{x}-V_{\psi} V_{y}\right)+A_{z x} V_{\psi} V_{\theta} \\
& -\left(a^{2} K_{f}+b^{2} K_{r}-M_{s} g h\right) \phi \\
& -\left(a^{2} B_{f}+b^{2} B_{r}\right) V_{f}+\left(a B_{f}-b B_{r}\right) V_{z} \\
& +\left(a K_{f}-b K_{r}\right) z \\
\dot{V}_{z} & =A_{x y} V_{\theta} V_{\phi}+a\left(F_{x f} \sin \delta_{f}+F_{y f} \cos \delta_{f}\right) \\
& -b\left(F_{x r} \sin \delta_{r}+F_{y r} \cos \delta_{r}\right) \\
+ & l\left(\Delta F_{x f} \cos \delta_{f}-\Delta F_{y f} \sin \delta_{f}\right) \\
+ & l\left(\Delta F_{x r} \cos \delta_{r}-\Delta F_{y r} \sin \delta_{r}\right)+I_{x z} \dot{V}_{\theta} \\
&
\end{aligned}\right.
$$

\section{Results and Discussion}

\subsection{Vehicle Model $M_{1}$ Validation}

The validity of the nonlinear vehicle models is examined using the available measured data reported in Ref. [18] under slalom maneuvers and with a constant speed. The relative performance of vehicle models $M_{1}$ and $M_{2}$ is further evaluated in terms of responses to acceleration/braking and steering inputs and with the vehicle speeds: 30,60 and $90 \mathrm{~km} \cdot \mathrm{h}^{-1}$. The number of cones used for the test is four cones and the distance between cones is $d=40 \mathrm{~m}$. The maximum lateral displacement is $y_{\max }=3.5 \mathrm{~m}$. The model $M_{1}$ simulations are performed with a front steering angle $\delta_{f}$ $=8^{\circ}$. Figs. $4 \mathrm{a}-4 \mathrm{c}$ present comparisons of the model $M_{1}$ responses to a slalom maneuvers in terms of longitudinal acceleration, lateral acceleration and yaw rate with the reported measured data. However, some differences between the simulation and measured data can also be observed. This may be attributed partially to the parameters and variables neglected in the modeling (six-DOFs), differences in the tires properties considered in the simulation model, inertias of sprung and unsprung mass and steering inputs used in the simulation model. The comparisons show good agreements between the simulation model responses and the measured data. So, the vehicle model $M_{1}$ obtained is good under considered slalom maneuvers.

\subsection{Comparisons of Two Models $M_{1}$ and $M_{2}$}

To compare the performance of the proposed two nonlinear vehicle models $M_{1}$ and $M_{2}$, different simulations are performed under slalom maneuvers and with vehicle speed $60 \mathrm{~km} \cdot \mathrm{h}^{-1}$.

Fig. 5 compares respectively the longitudinal velocity $V_{x}$ and the position of the vehicle in both models $M_{1}$ and $M_{2}$. The results show that the responses of longitudinal velocity and the vehicle position in the earth fixed frame $(0, x, y)$ have lower magnitudes in model $M_{1}$ than in model $M_{2}$.

Fig. 6 presents comparisons of the two models responses to slalom maneuver in term of roll and pitch angles and with vehicle speed $60 \mathrm{~km} \cdot \mathrm{h}^{-1}$.

The results clearly show that the roll response of the model $M_{1}$ is considerably lower compared with that of $M_{2}$. This is partially attributed to the higher roll moments of inertia and the added parameters in vehicle model $M_{1}$ as shown in Eq. (5). The peak pitch angle response of the first model $M_{1}$ is considerably lower compared with that of the model $M_{2}$.

Fig. 7 illustrates comparisons of the roll and pitch angles values of the vehicle models $M_{1}$ and $M_{2}$ with three different speeds $(30,60$ and 

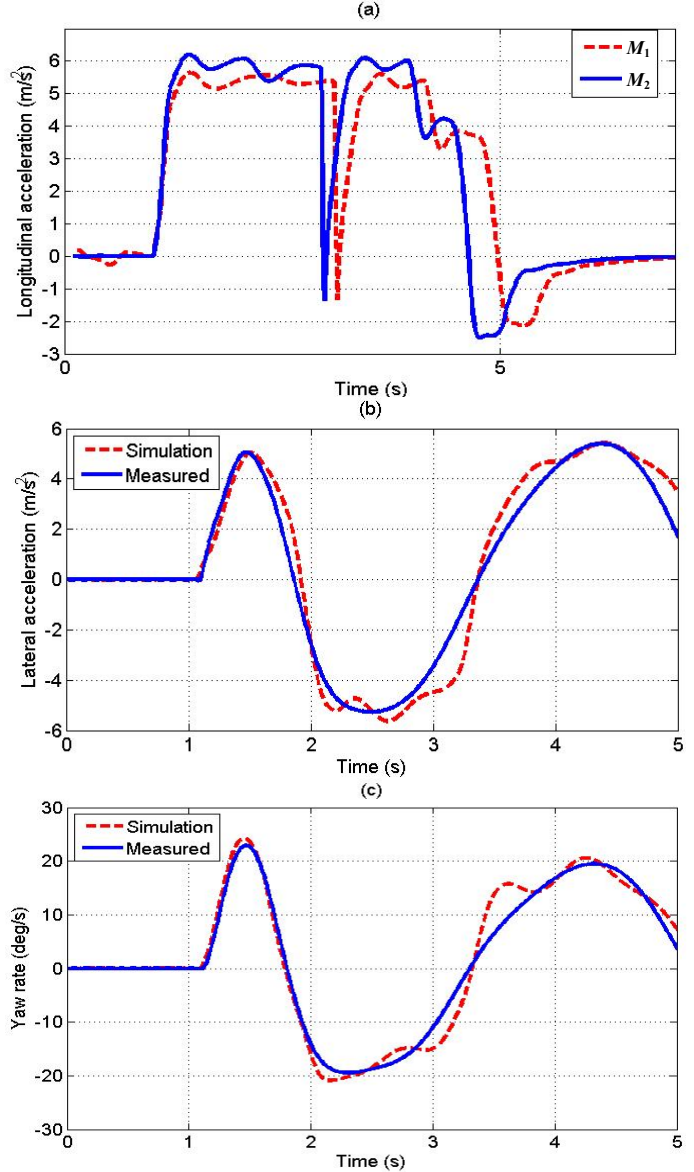

Fig. 4 Comparison of vehicle model $M_{1}$ during slalom maneuvering with the measured data reported in Ref. [18]: (a) longitudinal acceleration; (b) lateral acceleration and (c) yaw rate.

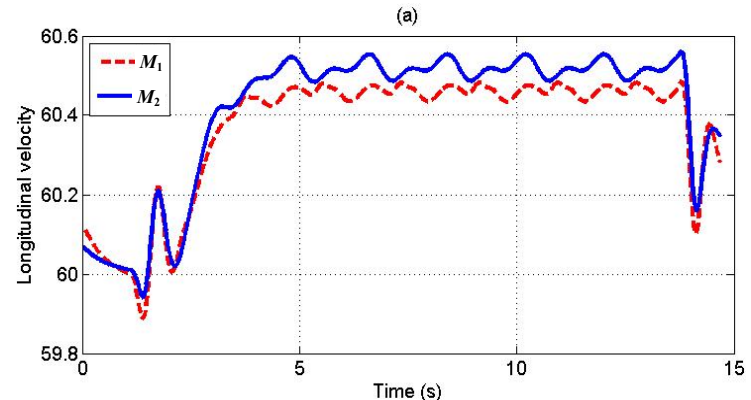

(b)

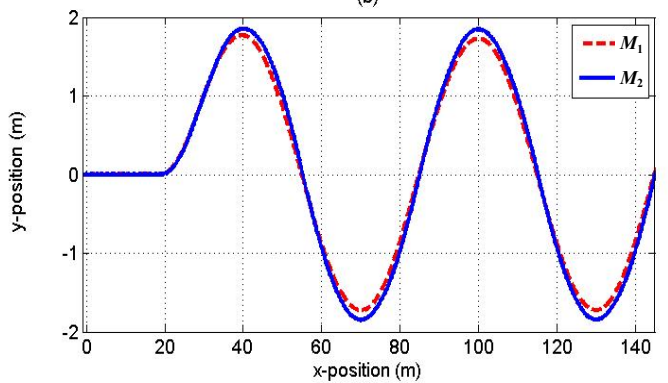

Fig. 5 (a) Longitudinal velocity in models $M_{1}$ and $M_{2}$ and (b) vehicle position in the earth fixed frame. 

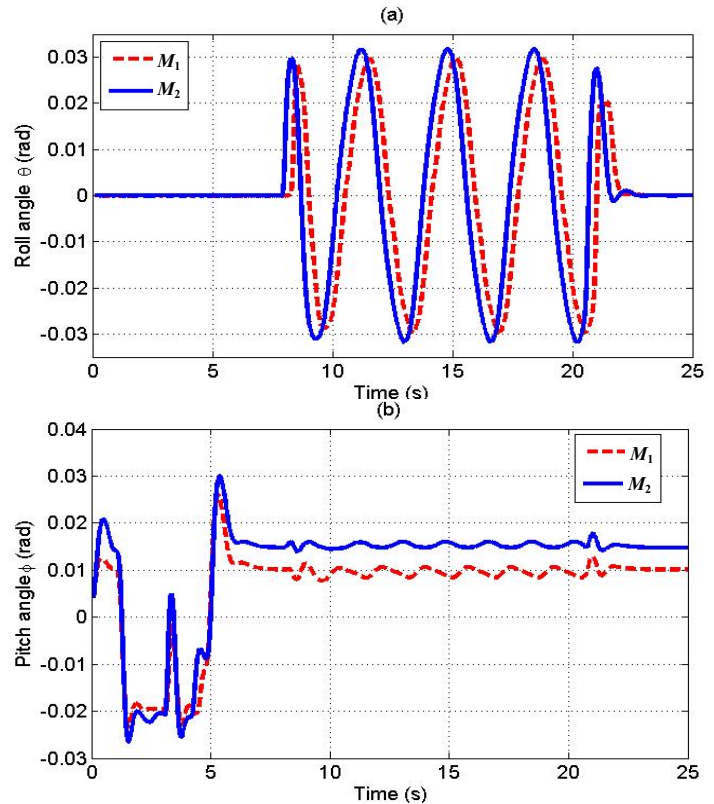

Fig. 6 (a) Roll angle and (b) pitch angle.

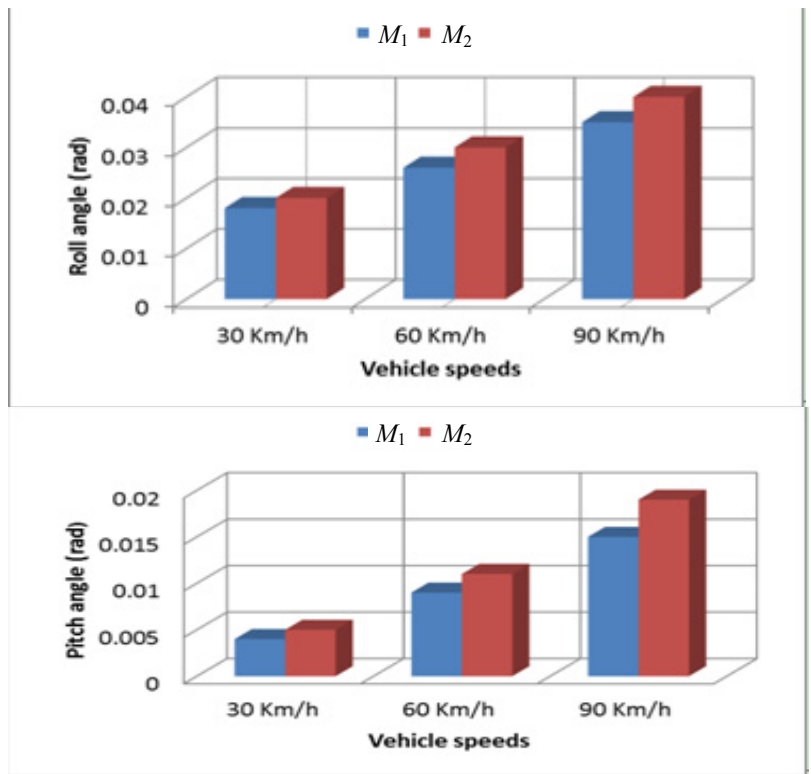

Fig. 7 Comparisons of the roll and pitch angles in models $M_{1}$ and $M_{2}$ under different speeds.

$\left.90 \mathrm{~km} \cdot \mathrm{h}^{-1}\right)$. The results show that the roll and pitch values of the model $M_{1}$ are considerably lower compared with those of the model $M_{2}$. The difference between the two models in terms of roll and pitch angles is relatively large when the vehicle speed increases. This may be partially attributed to the dynamic lateral and longitudinal load transfers which depend on the vehicle speed [13]. This means that the added parameters in the vehicle model $M_{1}$ tend to decrease the impacts of the load transfers and thus the magnitudes of vehicle roll and/or pitch motions.

\section{Conclusions}

This study investigated the potential benefits of such practical model $M_{1}$ on vehicle ride, handling, roll and pitch dynamic responses under various speeds and steering maneuvers. The dynamic responses of the proposed model $M_{1}$ are evaluated using generalized six-DOF nonlinear models. The roll, pitch, longitudinal and lateral dynamic performance are assessed under critical handling maneuvers. The simulation results show that the proposed model $M_{1}$ could yield considerably enhanced roll, pitch and yaw dynamics, handling performance as well as improved directional and stability driving of vehicle behavior. The practical benefits of the proposed model which takes CG of sprung mass, unsprung mass and total vehicle mass into account were further shown. The comparisons results of the proposed nonlinear vehicle models $M_{1}$ with the nonlinear model $M_{2}$ clearly demonstrate the potential advantages of model $M_{1}$ compared to $M_{2}$ in enhancing overall vehicle in terms of roll, pitch and directional responses. 


\section{References}

[1] Abbassi, Y., Ait-Amirat, Y., and Outbib, R. 2014. "Nonlinear Feedback Control and Trajectory Tracking of Vehicle." International Journal of Systems Science doi:10.1080/00207721.2014.880195.

[2] Cao, D. P., Rakhejab, S., and Su, C. Y. 2010. "Roll- and Pitch-Plane-Coupled Hydro-pneumatic Suspension. Part 2: Dynamic Response Analyses." Vehicle System Dynamics: International Journal of Vehicle Mechanics and Mobility 48 (4): 507-28.

[3] Ackermann, J. 1994. "Robust Decoupling, Ideal Steering Dynamics and Yaw Stabilization of 4WS Car." Automatica 30 (11): 1761-8.

[4] Abbassi, Y., Amirat, Y. A., and Outbib, R. 2011. "Nonlinear Feedback Control of Vehicle Speed." In Proceedings of the 18th IFAC (International Federation of Accountants) World Congress, 6279-84.

[5] Sayers, M. W. 1990. "Symbolic Computer Methods to Automatically Formulate Vehicle." Ph.D. thesis, University of Michigan.

[6] Heydinger, G., Bixel, R., Durisek, N., Yu, E., and Guenther, D. 1998. "Effects of Loading on Vehicle Handling." Presented at the SAE (Society of Automotive Engineers) International Congress and Exposition, Michigan, USA.

[7] Whitehead, R., Travis, W., Bevly, D., and Flowers, G. 2004. "A Study of the Effects of Various Vehicle Properties on Rollover Propensity." Presented at the SAE Automotive Dynamics, Stability \& Controls Conference and Exhibition, Michigan, USA.

[8] Hac, A. 2005. "Effects of Brake Actuator Error on Vehicle Dynamics and Stability." Presented at the SAE 2005 World Congress \& Exhibition, Michigan, USA.

[9] Hegazy, S., Rahnejat, H., and Hussain, K. 2000. "Multi-body Dynamics in Full Vehicle Handling Analysis under Transient Maneuver." Vehicle System Dynamics: International Journal of Vehicle Mechanics and Mobility 34 (1): 1-24.

[10] Cao, D. P., Rakheja, S., and Su, C. Y. 2010, "Roll- and Pitch-Plane Coupled Hydro-pneumatic Suspension." Vehicle System Dynamics: International Journal of Vehicle Mechanics and Mobility 48 (3): 361-86.

[11] Shim, T., and Ghike, C. 2007. "Understanding the Limitations of Different Vehicle Models for Roll Dynamics Studies." Vehicle System Dynamics: International Journal of Vehicle Mechanics and Mobility 45 (3): 191-216.

[12] Abbassi, Y., Ait-Amirat, Y., and Outbib, R. 2007. "A Global Model for Vehicle Dynamics." In Proceeding of AVCS (Advanced Vehicle Control Systems), 285-90.

[13] Pacejka, H. B. 2005. Tire and Vehicle Dynamics. 2nd ed.. United Kingdom: Butterworth-Heinemann.

[14] Wong, J. Y. 2006. Theory of Ground Vehicles. 3rd ed.. New York: Wiley.

[15] Klasson, J. 2002. “A Generalized Crosswind Model for Vehicle Simulation Purposes." Vehicle System Dynamics Supplement 37: 350-9.

[16] Novel, B., and Chou, H. 2005. "Global Vehicle Control Using Differential Braking Torques and Active Suspension Forces." Vehicle System Dynamics 43 (4): 261-84.

[17] Li, L., Wang, F. Y., and Zhou, Q. Z. 2006. "Integrated Longitudinal and Lateral Tire/Road Friction Modeling and Monitoring for Vehicle Motion Control." IEEE Transactions on Intelligent Transportation Systems 7 (1): 1-19.

[18] Bernard, J. E., Winkler, C. B., and Fancher, P. S. 1973. A Computer Based Mathematical Method for Predicting the Directional Response of Trucks and Tractor-Trailers. UMTRI technical report UM-HSRI-PF-73-1, The University of Michigan, USA. 